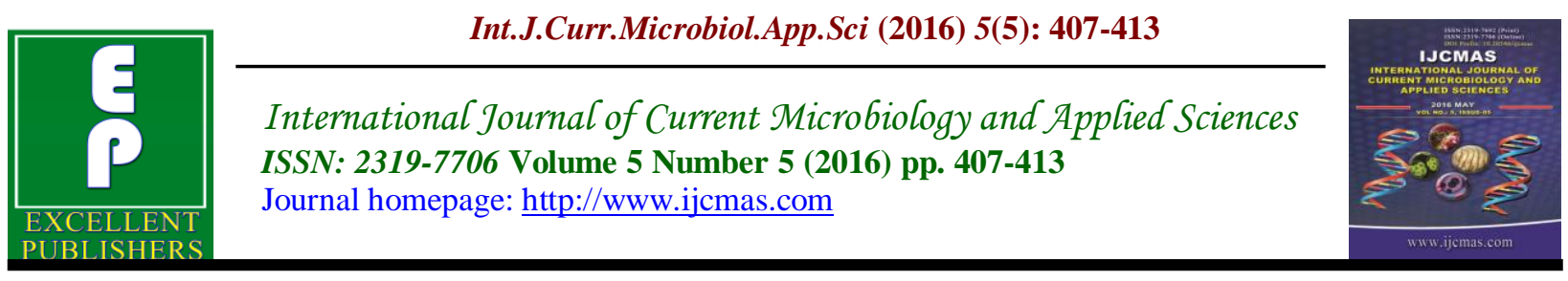

Original Research Article

http://dx.doi.org/10.20546/ijcmas.2016.505.042

\title{
Rhamnolipid Biosurfactant Production by Strain of Pseudomonas aeruginosa using Different Raw Materials
}

\author{
S. N. Zodpe* \\ P.G. Department of Microbiology, Shri Shivaji College of Arts, Commerce \& Science, \\ Akola 444003 M.S, India \\ *Corresponding author
}

Keywords

Biosurfactant, Rhamnolipid, Pseudomonas aeruginosa, Raw materials.

\section{Article Info}

Accepted:

15 April 2016

Available Online:

10 May 2016
A B S T R A C T

Microbial surfactants are amphiphilic compounds. The unique properties of biosurfactant allow their use and possible replacement of chemically synthesized surfactant. The composite soil samples from different oil mills were screened for the presence of Pseudomonas species. The four confirmed strain of Pseudomonas show positive results and were further studied for the presence of surfactant production ability using five different medium. The result of surfactant production was expressed in terms of Rhamnose.

\section{Introduction}

Biosurfactants are amphiphilic compounds which are produced on living surfaces, mainly on surfaces of microorganisms or may also secreted extracellularly and it contains both hydrophilic and hydrophobic moieties which reduces the surface and interfacial tension of the surface and interface respectively. Since biosurfactant and bioemulsifiers both exhibit emulsification properties, bioemulsifiers are frequently considered with biosurfactant, even though emulsifiers may not lower surface tension. A biosurfactant can have one of the following structures: glycolipids, mycolic acid, polysaccharide-lipid composite, lipoprotein/ lipopeptide, phospholipid, or the microbial cell surface itself.
Significant attention has been given in the past to the synthesis of surface-active molecules from biological source because of their potential use in food-processing, oil industry, and pharmacology. Even though the type and quantity of the microbial surfactants produced depends mainly on the producer organism, factors like nitrogen and carbon, temperature, aeration and trace elements also affect their production by the organism. The major classes of biosurfactant includes glycolipids, lipopeptides and lipoproteins, fatty acids, phospholipids, neutral lipids and polymeric microbial surfactants. Rhamnolipid biosurfactants are surface active compounds produced by Pseudomonas aeruginosa. They can reduce 
surface tension, stabilise emulsions, promote and are generally non-toxic, non-hazardous and biodegradable. Due to their diversity, environmentally friendly nature, possibility of large scale production, selectivity, effectiveness under extreme conditions in small quantities, production on renewable sources and potential applications in environmental protection. Biosurfactants are gaining prominence over chemical surfactants. In addition, the valuable carbohydrates moiety, rhamnose of the Rhamnolipid biosurfactant is used for transport of insoluble drugs in humans and acts as precursor for high quality flavour components.

\section{Materials and Methods}

The study on production of surfactant of microbial origin using Pseudomonas aeruginosa was conducted by adopting different materials and methods for experimentations.

\section{Collection of Samples}

\section{Isolation of Surfactant Producing Pseudomonas Species}

\section{Isolation of Pseudomonas aeruginosa}

The plates containing selective medium Cetrimide agar was inoculated with hydrocarbon rich soil collected from petrol pump and garage. $1 \mathrm{gm}$ of soil sample was added to $10 \mathrm{ml}$ distilled water and vortexed thoroughly. The supernatant was serially diluted and plated on selective medium i.e. Cetrimide agar. All the plates were then incubated at $30^{\circ} \mathrm{C}$ for 48 hours. After incubation, cultures were examined for identification.

\section{Identification of Pseudomonas Species}

\section{Cultural Characteristics}

The isolated colonies of Pseudomonas species from Cetrimide agar was inoculated separately on blood agar as well as on MacConkey agar and was further identified on the basis of heamolysis and colony characters.

\section{Biochemical Test}

The colonies showing haemolysis were further subcultured on nutrient agar slant. The strains were then studied for its biochemical characterization by adopting standard method for IMViC, Urease, Oxidase, Gelatinase and Nitrate Reductase. All the tentatively confirmed isolates of Pseudomonas species were further screened for biosurfactant production ability.

\section{Screening of Surfactant Producing Pseudomonas}

The screening of biosurfactant was done by adopting the phenol sulphuric acid and Erythrocyte Haemolysis method respectively.

Phenol Sulphuric Acid Method :- In phenol sulphuric acid method $1 \mathrm{ml}$ of $5 \%$ phenol was added to the supernatant and to this added $5 \mathrm{ml}$ of conc. Sulphuric acid drop by drop the colour changes from yellow to orange indicates the biosurfactant production.

Erythrocyte Haemolysis Method :- In this method, to the $10 \mathrm{ml}$ of supernatant Zinc Chloride was added and precipitation appearance was seen. To this precepitation $10 \mathrm{ml}$ of sodium phosphate buffer was added and extracted it with diethyl ether and allow to evaporate to dryness after dryness, powder was remain and the powder was mixed with $100 \mathrm{ml}$ of methanol from that solution $10 \mu$ was spotted on filter paper disc and kept it on blood agar plates. Plates were incubated at room temperature for 2 days. Zone of Haemolysis indicates positive test. 
Production of biosurfactant by isolated Pseudomonas species :-

Biosurfactant production was carried out utilising five different Raw materials i.e. Soybean oil, Olive oil, Whey, Glucose, Mannitol.

All the medium were sterilised in autoclave at $121^{\circ} \mathrm{C}$ for 15 minutes at $15 \mathrm{lbs}$ pressure. The surfactant producing strain of Pseudomonas was aseptically inoculated in each medium. Uninoculated set for each medium was maintained as a control set.The inoculate medium were further incubated on rotary shaker at room temperature for about 2 days.

\section{Extraction of Biosurfactant}

The extraction of biosurfactant was done by subjecting the enriched broth to centrifuge at $3000 \mathrm{rpm}$ for 30 minutes. The supernatant thus obtained was separated using micropipette and further use to estimate the amount of surfactant produced. Simultaneously the optical density of enriched culture was measured at $540 \mathrm{~nm}$ on eighth day of incubation. The uninoculated nutrient broth set was maintained as control.

\section{Quantative analysis by Biosurfactant}

\section{Estimation of Rhamnose}

\section{Spectrophotometric Methods}

One of the most widely used methods for RL quantification is the orcinol test. It consist of heating the solvent obtained extracts of culture supernatant in the presence of sulphuric acid and orcinol (1, 3dihydroxy-5-methylbenzene). The rhamnose groups of RL are hydrolyzed and transformed into methyl furfural, which then reacts with the orcinol to produce a bluegreen colour, that can be measured spectrophotomatically at $421 \mathrm{~nm}$ (Chandrasekharan and Be Miller, 1980; Koch et al., 1991)

A standard curve is prepared with rhamnose or preferably with a standard RL mixture for quantification

\section{Result and Discussion}

Rhamnolipid are the best studied glycolipids having excellent surface activity. Their utilization in various application areas of environment, health, food, cosmetics, oil industries, etc. have made it the potential candidates that could replaced the chemically synthesized surfactants because these are derived from the natural sources in a pure form and they have a low toxicity levels.

The production of Rhamnolipid by Pseudomonas aeruginosa was confirmed by two methods - Phenol Sulphuric Acid Method and Erythrocytes Blood Haemolysis Method. The Aim of this work is production of Rhamnolipid using different raw materials such as soybean oil, olive oil, whey, glucose and mannitol as a carbon sources. Among 15 oil degrading bacterial isolates from hydrocarbon polluted environments four strains of Pseudomonas aeruginosa were found to be effectively utilized soybean and olive oil for biosurfactant production. The detailed study of morphological and biochemical characterization of the isolates showed in table 2 and 3 . That these isolates were obtained from various soil samples and were different strains of Pseudomonas aeruginosa and were designated as P1, P2, P3 and P4. Mineral salt medium was used for growing biosurfactant followed by the addition of various substrates like soybean oil, olive oil, 
whey, glucose and mannitol to test their influence on biosurfactant production shown in table 4 and figure 3. Among the five substrates tested $\mathrm{P} 1$ produced a maximum of $0.342 \mathrm{gm} / \mathrm{L}$ Rhamnolipid with soybean oil followed by olive oil (0.130), whey (0.092), glucose (0.120) and mannitol (0.064) gm/l at $550 \mathrm{~nm}$. This result correlates with K. S. M. Rhaman et al., obtained $4.3 \mathrm{~g} / 1$ Rhamnolipid was produced from $6 \mathrm{~g} / \mathrm{l}$ of soybean oil which was higher when compared to $0.625 \mathrm{gm}$ Rhamnolipid per soybean oil. This result is also in accordance with Lang and Wullbrandt, they revealed about 2.2-5.8 $\mathrm{g} / 1(10 \%-20 \%)$ of Rhamnolipid production using 20-30 g/l of soybean oil by two different strains of Pseudomonas aeruginosa. This result similar with K.S.M. Rahman et al., showed higher biosurfactant productions with soybean oil than glycerol. They found out that increase in biosurfactant production even after the bacterial growth had reached stationary phase. This result also similar with Abalos and co-workers (2001), have reported the used of soybean oil as a carbon sources for the production of new Rhamnolipid by Pseudomonas aeruginosa. Further the study with P2 isolate showed maximum biosurfactant production of $0.130 \mathrm{gm} / \mathrm{L}$ Rhamnolipid with olive oil followed by soybean oil, whey, glucose and mannitol at $550 \mathrm{~nm}$. This results correlates with Haba and co-workers (2000), compared the composition of used olive oil and sunflower oils with the standard unused oils and they found out that the most important difference in the used oil is the presence of $22.52 \%$ of fatty acid of low chain length. After tested by Pseudomonas strain they showed stastisfactory growth when cultivated on used olive oil. This results also similar with Vollbrecht and coworkers (1999), they used domestic vegetable oil for production of biosurfactants by Tsukamurella species
DSM44370 they tested several vegetable oil and the best result were achieved with oleic acid rich and rapeseed oil. Further study with P3 isolate showed maximum biosurfactant production of $0.236 \mathrm{gm} / \mathrm{L}$ Rhamnolipid with whey followed by soybean oil (0.206), olive oil (0.107), glucose (0.198) and mannitol (0.07) gm/l at $550 \mathrm{~nm}$. This results correlates with Aylin Kitlik Colak and Huseyin Kahraman they produced Rhamnolipid by a wild type and recombinant strain of Pseudomonas aeruginosa using whey as a carbon source and they showed Rhamnolipid accumulation started after the cells reached stationary phase, and significance differences in production between the strains depending on cultivation conditions. This result is also in accordance with Koch and co-workers (1988), has developed a strain of Pseudomonas aeruginosa to used whey for the production of Rhamnolipid and Bacillus subtilis is also known to produce surfactin using whey as a substrate. This result also similar with Aparnaa et al., (2012), they reported that Rhamnolipid is "primary metabolite" when whey used as a carbon source its production coincides the exponential growth phase. Similar results was obtained from Silva et al., (2010), when whey used as a carbon source the Rhamnolipid production was 2.7,1.85,1.78 and $0.24 \mathrm{~g} / \mathrm{l}$. Further study with P4 isolates showed maximum biosurfactant production of $0.342 \mathrm{gm} / \mathrm{L}$ Rhamnolipid with Glucose followed by soybean oil, olive oil, whey and mannitol at $550 \mathrm{~nm}$. This results correlates with Praveesh B. V. et al., 2011, they stated that maximum amount of Rhamnolipid was produced when $3 \%$ of whey was used as a carbon source. This result is also in accordance with M. Irfan Maqsood and Asif Jamal 2011 they reported that Rhamnolipid concentration decreased sharply when glycerol concentration was about 6-7\%. 
Table.1 Morphological Characteristic of Pseudomonas species

\begin{tabular}{|l|c|c|c|c|}
\hline Cultural characteristics & Isolate No.1 & Isolate No.2 & Isolate No.3 & Isolate No.4 \\
\hline Size & $1-2 \mathrm{~mm}$ & $1-2 \mathrm{~mm}$ & $1 \mathrm{~mm}$ & $1 \mathrm{~mm}$ \\
\hline Shape & circular & circular & circular & Circular \\
\hline Margin & Irregular & Irregular & Irregular & Irregular \\
\hline Elevation & Umbonate & Umbonate & Umbonate & Umbonate \\
\hline Opacity & Opaque & Opaque & Opaque & Opaque \\
\hline Motility & Motile & Motile & Motile & Motile \\
\hline Gram Characters & -ve & -ve & -ve & -ve \\
\hline
\end{tabular}

Table.2 Biochemical characteristics of Isolates strains

\begin{tabular}{|c|c|c|c|c|}
\hline Test perfom & Isolate No.1 & Isolate No. 2 & Isolate No.3 & Isolate No.4 \\
\hline \multicolumn{5}{|l|}{ Fermentation of Sugars. } \\
\hline Glucose & $+\mathrm{ve}$ & $+\mathrm{ve}$ & $+\mathrm{ve}$ & $+\mathrm{ve}$ \\
\hline Mannitol & -ve & -ve & -ve & -ve \\
\hline Lactose & -ve & -ve & -ve & $-\mathrm{ve}$ \\
\hline Sucrose & -ve & -ve & -ve & -ve \\
\hline \multicolumn{5}{|l|}{ IMViC Test. } \\
\hline Indole & -ve & -ve & -ve & -ve \\
\hline Methyl Red & $-\mathrm{ve}$ & $-\mathrm{ve}$ & -ve & -ve \\
\hline Voges prausker & -ve & -ve & -ve & -ve \\
\hline Citrate & $+\mathrm{ve}$ & $+\mathrm{ve}$ & $+\mathrm{ve}$ & $+\mathrm{ve}$ \\
\hline \multicolumn{5}{|l|}{ Enzyme Test } \\
\hline Catalase & $+\mathrm{ve}$ & $+\mathrm{ve}$ & $+\mathrm{ve}$ & $+\mathrm{ve}$ \\
\hline Oxidase & $+\mathrm{ve}$ & $+\mathrm{ve}$ & $+\mathrm{ve}$ & $+\mathrm{ve}$ \\
\hline Amylase & -ve & -ve & -ve & -ve \\
\hline Gelatinase & $+\mathrm{ve}$ & $+\mathrm{ve}$ & $+\mathrm{ve}$ & $+\mathrm{ve}$ \\
\hline Urease & -ve & -ve & -ve & -ve \\
\hline \multicolumn{5}{|c|}{$\begin{array}{l}\text { On the basis of cultural, biochemical characteristics and enzyme study, following isolates were } \\
\text { confirmed }\end{array}$} \\
\hline $\begin{array}{l}\text { Tentatively confirmed } \\
\text { Isolate }\end{array}$ & $\begin{array}{l}\text { Pseudomonas } \\
\text { aeurignosa }\end{array}$ & $\begin{array}{l}\text { Pseudomonas } \\
\text { aeurignosa }\end{array}$ & $\begin{array}{l}\text { Pseudomonas } \\
\text { aeurignosa }\end{array}$ & $\begin{array}{l}\text { Pseudomonas } \\
\text { aeurignosa }\end{array}$ \\
\hline
\end{tabular}

Whereas $:-+v e=$ Positive,- ve $=$ Negative

Fig.1 Rhamnolipid Production by using Raw materials

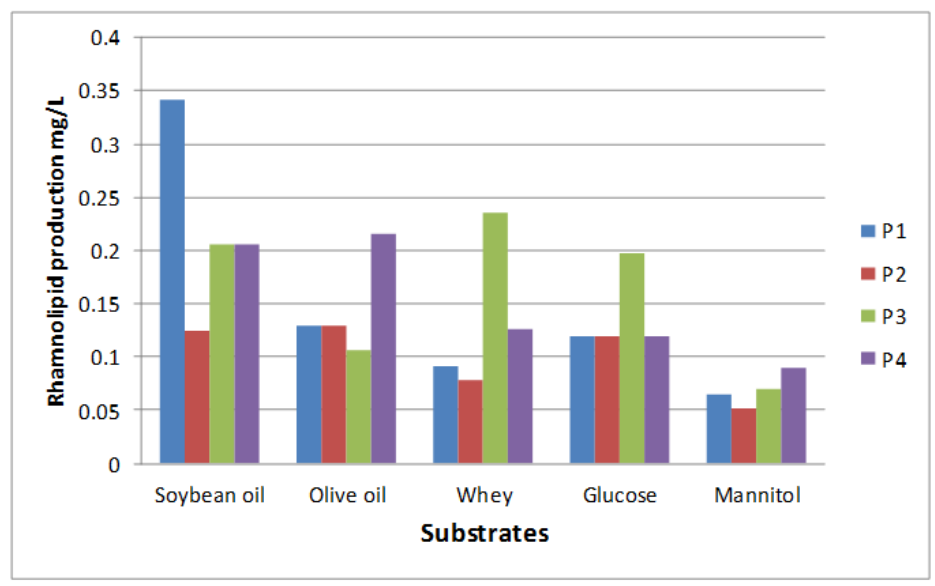


Fig.2 Graphical representation of Rhamnolipid production by different Raw Materials

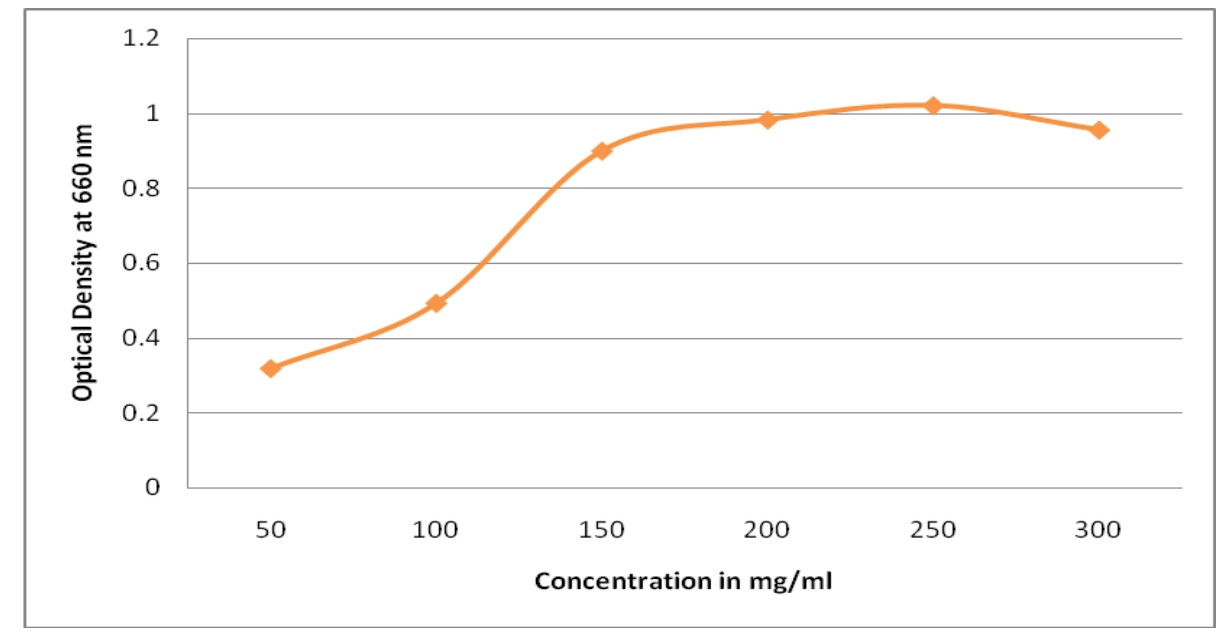

It was reported that highest concentration i.e. $2 \mathrm{~g} /$ lit was observed at $3 \%$ of glycerol concentration. However, Tugba Subasioglu and Emir Cansunar 2008, reported that production of biosurfactant was relatively high when glucose was the carbon source. In addition Rhamnolipid concentration reached at its maximum level when mannitol was the carbon source. The effect of glucose on Rhamnolipid production was also shown by Ugur SIDAL and Ebru Sebnem YILMAZ 2012, they reported that maximum biosurfactant concentration $(387.5 \mathrm{mg} /$ lit $)$ was obtained at an initial glucose concentration of $2 \%$ and also reported that higher and lower concentration inhibit Rhamnolipid production.

In above study the five different substrates were used for maximum production of Rhamnolipid i.e. soybean oil, olive oil, whey, glucose and mannitol. And the maximum Rhamnolipid was produced when soybean oil used as carbon source. Hence further study i.e. rhamnose estimation was continued with P1 isolate, which produce highest Rhamnolipid $0.342 \mathrm{gm} / \mathrm{l}$ by soybean oil. All microorganisms required for growth a source of carbon hydrogen, nitrogen, oxygen and to a smaller degree sulfur and phosphorus. These materials are available in many forms. The choice of raw materials is very important to the overall economics of the process. The carbon source is very important in biosurfactant production and the wide variety has been used. They include hydrocarbon, carbohydrate and vegetable oil sources. Some organisms produced biosurfactant only in hydrocarbon, other only in carbohydrates and a still other used several substance, in combination or separately.

In conclusion, the present investigation results indicated that the four different strains of Pseudomonas aeruginosa isolated during study were efficient in Rhamnolipid biosurfactant production. Soybean oil was found to produce maximum Rhamnolipid production to several folds than olive oil and whey. This results suggested the best use of low cost renewable carbon sources like soybean oil, olive oil and whey for the biosurfactant production, which could be applied for bioremediation of hydrocarbon contaminated sites and also in enhanced oil recovery

\section{References}

Abalos, A., Pinazo., A., Infante, M.R., Casals, M., Garcia, F., Manresa, A. 
2001. Langmvir, 17: 1367-1371.

Aparnaa, A., Srinikethana, G., Smitha, H. 2012. Production and Characterization of biosurfactant produced by a novel Pseudomonas Species.2B. Colloid surface B. 95: 23-29.

Aylin Kitlik Colak, Huseyin Kahraman 2013. The use of raw cheese whey and olive oil mill wastewater for Rhamnolipid production by recombinant Pseudomonas aeruginosa, J. Environ. Experimental Biol., 11: 125-130.

Chandrasekaran, E.V., Be Miller, J.N. 1980. In: whistler RL (ed) Methods in carbohydrate chemistry. Academic Press, New York, p89.

Haba, E., Espuny, M.J., Busquets, M., Manresa, A. 2000. Screening and production of Rhamnolipid by Pseudomonas aeruginosa 47T2 NCIB from waste frying oils. J. Appl. Microbiol., 88: 379-387.

Rahman, K.S.M., Thahira, J., Rahman, Stepten McClean, Roger Marchant, Ibrahim M., Banat. 2000. Rhamnolipid biosurfactants production by strains of Pseudomonas aeruginosa using low coast raw materials, Biotechnology research group, coleraine-BT521SA.
Koch, A., Resier, K.J., Kapelli, O., Fiechter, A. 1988. Genetic construction of lactose-utilizing strains of Pseudomonas aeruginosa and their application in Biosurfactants production. Biotechnol., 6: 1335-1339.

Lang, S., Wullbrant, D. 1999. Appl. Microbiol. Biotechnol., 51 : 22-32.

Maqsood, M., Irfan, Asif Jamal. 2011. Factors affecting the Rhamnolipid biosurfactant production. Pak .J. Biotechnol., Vol.8(1) 1-5.

Praveesh, B.V., Soniyamby, A.R., Mariappan, C., Kauithankumari, P., Palaniswamy, M., Lalitha, S. 2011. Biosurfactant production by Pseudomonas species from soil using Whey as a carbon source. New York Sci. J., 4(4).

Silva, S.N.R.L., Fariasb, C.B.B., Rufinob, R.D., Lunab, J.M., Sarubbo, L.A. 2010. Glycerol as substrates for the production of biosurfactant by Pseudomonas aeruginosa UCT0992. Colloid. Surface B, 79: 174-183.

Ugur Sidal, Ebru Sebnem Yilma. 2012. Production of Rhamnolipid (A Biosurfactant) using free and immobilized cells Pseudomonas species. Kafkas Univ. Vet. Fak. Derg., 18(2): 285-289.

\section{How to cite this article:}

Zodpe, S.N., 2016. Rhamnolipid Biosurfactant Production by Strain of Pseudomonas aeruginosa using Different Raw Materials. Int.J.Curr.Microbiol.App.Sci.5(5): 407-413. doi: http://dx.doi.org/10.20546/ijcmas.2016.505.042 